\title{
61. Typen und Konzepte des Spracherwerbs
}

1. Einleitung

2. Komponenten des Spracherwerbs

3. Der Prozess des Spracherwerbs

4. Die wesentlichen Theorien

5. Abschließende Bemerkung

6. Literatur in Auswahl

\section{Einleitung}

$\mathrm{Zu}$ den verschiedenen Gaben, mit denen die List der Natur den Menschen bedacht hat, zählt auch die Fähigkeit, eine Sprache zu erlernen und $\mathrm{zu}$ gebrauchen: ein jeder eignet sich als Kind eine Sprache, die Muttersprache, an und macht bald mit unterschiedlichem Glück und Geschick Gebrauch von ihr. Zwar ist dieser Prozess nicht, wie gelegentlich angenommen wird, schnell und mühelos. Er erstreckt sich über viele Jahre. Dies ist offen- kundig für den Bereich des lexikalischen Erwerbs: neue Wörter oder auch nur neue Gebrauchweisen bekannter Wörter lernt man das ganze Leben. Es gilt aber durchaus auch für strukturelle Eigenschaften, etwa aus der Syntax oder der Morphologie. Selbst Achtbis Zehnjährige beherrschen manche Konstruktionen ihrer Sprache noch nicht. Dennoch: mit der Pubertät ist dieser Teil des Erstspracherwerbs im Wesentlichen abgeschlossen, und das Ergebnis ist in aller Regel eine ,perfekte“ Beherrschung der Sprache. Mit ,perfekt" ist hier nicht gemeint, dass das Sprachvermögen schon seinen denkbaren Höhepunkt erreicht hätte; nicht jeder Deutsche schreibt mit vierzehn Jahren wie Goethe mit vierzig; gemeint ist, dass ein jeder normale Mensch etwa mit der Pubertät über eine Sprachbeherrschung verfügt, die sich von je- 
ner seiner sozialen Umgebung nicht auffällig unterscheidet. In diesem Sinne ist also die Sprachbeherrschung eines Hilfsarbeiters mit einem Wortschatz von 500 aktiv gebrauchten Wörtern und schlichter, aber klarer Syntax ,perfekt', nicht hingegen die Sprachbeherrschung eines ausländischen Goetheforschers mit 5000 aktiv gebrauchten Wörtern und höchst komplexer, wenn auch bisweilen falscher Syntax und merklichem Akzent. In einem Satz: der Erstspracherwerb ist ein natürlicher Prozess, er wird nicht systematisch und planvoll von außen gesteuert, dauert recht lange und führt in der Regel zur ,perfekten“ Beherrschung der zu lernenden Sprache.

Die meisten lernen eine zweite, viele eine dritte oder vierte Sprache. Verlauf und Endergebnis des Zweitspracherwerbs (oder "Mehrspracherwerbs") sind, verglichen mit dem Erstspracherwerb, relativ uneinheitlich. Dafür sind viele Faktoren verantwortlich, von denen zwei besonders wichtig sind: Alter und Art des Erwerbs variieren beträchtlich. Der Erstspracherwerb setzt praktisch mit der Geburt ein (es gibt sogar Vorstellungen, dass schon pränatale Einflüsse eine Rolle spielen). Der Zweitspracherwerb beginnt zu sehr unterschiedlichen Zeitpunkten. Zum Ersten ist möglich, dass von Anfang an zwei Sprachen gelernt werden - ein Fall, den man sinnvollerweise nicht als ,Zweitspracherwerb", sondern als ,bilingualen Erstspracherwerb" bezeichnet (Meisel 1994). Eine zweite Sprache kann aber zu jedem beliebigen späteren Zeitpunkt hinzutreten, im frühen Kindesalter, wenn kaum die ersten Strukturen der Muttersprache vorhanden sind, bis zum fortgeschrittenen Erwachsenenalter, in dem das Lernvermögen nicht nur für die Sprache nach allgemeiner Erfahrung nachgelassen hat: der Übergang ist daher gleitend. Das Lernalter führt, so sollte man zumindest annehmen, zu verschiedenen Formen des Zweitspracherwerbs. Ebenso wichtig ist aber die besondere Art des Erwerbs. Der Erstspracherwerb erfolgt in der alltäglichen Kommunikation, ohne systematisch steuernde Intervention von außen. Kinder haben zwar ein außerordentlich feines Ohr für sprachliche Eigenschaften, aber sie sind gegenüber korrigierenden Einflüssen auf ihre Art zu reden zumindest bis ins Schulalter sehr widerborstig. Sie verlassen sich nicht nur in dieser Hinsicht lieber auf das, was sie selber hören, als auf das, was man sie heißt. Der Zweitspracherwerb kann in derselben Weise, nämlich in der alltäglichen Kommunikation, erfolgen - etwa bei Kindern, die mit geringer zeitlicher Verzögerung eine zweite Sprache lernen, weil ihre soziale Umgebung dies erfordert, oder beim Spracherwerb ausländischer Arbeiter im Erwachsenenalter. Er kann aber auch das Produkt einer systematischen Intervention, also des Sprachunterrichts, sein, und je nach Art dieses Unterrichts wiederum sehr unterschiedliche Formen annehmen. Dementsprechend spricht man von ungesteuertem und gesteuertem Zweitspracherwerb; für letzten sagt man oft auch Fremdspracherwerb. Je nach Alter und nach Art des Unterrichts gibt es hier wiederum sehr unterschiedliche Unterformen. Wenn man daher von „Typen“ des Spracherwerbs redet, so muss man sich vor Augen halten, dass es sich um ein reiches Spektrum von Erscheinungsformen handelt, die gleitend ineinander übergehen und unter denen die eine oder andere besonders häufig, aus praktischen Gründen besonders wichtig oder unter wissenschaftlichen Gesichtspunkten besonders aufschlussreich ist. Drei in diesem Sinne ,typische“ Exponenten sind

- der monolinguale Erstspracherwerb; er ist insofern von besonderer Bedeutung, weil jeder normale Mensch eine erste Sprache lernt; so liegt denn die Annahme nahe, dass er am reinsten die naturgegebenen Gesetzlichkeiten des menschlichen Sprachvermögens widerspiegelt;

- der ungesteuerte Zweitspracherwerb im Erwachsenenalter, wie wir ihn bei ausländischen Arbeitern beobachten; nicht jeder lernt im Erwachsenenalter eine zweite Sprache, und insofern ist die Form des Spracherwerbs weniger „natürlich“; sie ist es aber insofern, als sie ganz von naturgegebenem Umgang mit neuem sprachlichem Material bestimmt ist und nicht zugleich die Auswirkungen einer gezielten, mehr oder minder sinnvollen Intervention - eben des Unterrichts - reflektiert;

- der traditionelle grammatikorientierte Fremdspracherwerb in der Schule, wie wir ihn in besonders ausgeprägter Form im klassischen Lateinunterricht finden; kein Mensch lernt von Natur aus eine Sprache auf diese Weise; aber er kann sie auf diese Weise lernen, und in vielen Fällen, wie eben im Lateinunterricht, ist es sogar die einzige Möglichkeit.

Diese drei Ausprägungen des Spracherwerbs haben charakteristische Gemeinsamkeiten und Verschiedenheiten, unter denen die folgenden besonders augenfällig sind: 
A. Zweitspracherwerb und Fremdspracherwerb ist gemeinsam, dass (a) der Lerner bereits eine Sprache beherrscht, (b) er im Alter vorangeschritten ist, und (c) das Ergebnis in aller Regel nicht, wie beim Erstspracherwerb, eine ,perfekte' Beherrschung der zu lernenden Sprache ist (obwohl dies auch wiederum nicht ausgeschlossen ist).

B. Erstspracherwerb und Zweitspracherwerb sind gleichsam ,natürliche“" Prozesse, d. h. sie beruhen auf den uns von der Natur gegebenen Mechanismen der Sprachverarbeitung und den Prinzipien, die sie steuern. Der Fremdspracherwerb ist demgegenüber ein Versuch, auf diesen Prozess auf Grund gewisser Überlegungen oder auch praktischer Erfahrungen steuernd von außen einzuwirken, um ihn so zu optimieren.

C. Der Erstspracherwerb ist nur eine Komponente in der gesamten kognitiven und sozialen Entwicklung des Kindes; bei Zweitspracherwerb wie bei Fremdspracherwerb ist diese Entwicklung hingegen in wesentlichen Teilen abgeschlossen.

Nun sind, wie gesagt, diese drei Formen Extremfälle, zwischen denen es zahlreiche Zwischenstufen gibt - etwa den bilingualen Erstspracherwerb (weltweit gesehen vielleicht sogar der häufigere Fall als der monolinguale Erstspracherwerb), den Zweitspracherwerb im Kindesalter, den Fremdspracherwerb im kommunikativ orientierten, vielleicht gar monolingualen Unterricht, usw. usw. Ein weiterer Grenzfall ist der „Wiedererwerb“ einer Sprache, die einmal gelernt worden war, dann aber mehr oder minder vergessen wurde.

Es ist nach all dem kein Zufall, dass es in der Forschung keine einheitliche Terminologie für die verschiedenen Formen des Spracherwerbs gibt. Sinnvoller als der Versuch, feste "Typen" voneinander abzugrenzen, ist daher die Frage, welche Züge den unterschiedlichen Formen des Spracherwerbs gemeinsam sind und in welchen sie sich unterscheiden. Dies wollen wir im folgenden Abschnitt systematisch betrachten.

\section{Komponenten des Spracherwerbs}

Der Spracherwerb gleich welcher Art ist immer ein sehr komplexer Prozess, der sich beim Kind wie beim Erwachsenen über viele Jahre erstreckt und dessen Verlauf wie dessen Endergebnis von einer Reihe von interagierenden Faktoren bestimmt wird. Bei aller
Unterschiedlichkeit im Einzelnen müssen jedoch immer drei Komponenten gegeben sein. Der Lerner muß über ein bestimmtes, im Gehirn gespeichertes Sprachlernvermögen verfügen, das wiederum ein Teil seines angeborenen und im Laufe des Lebens entfalteten Sprachvermögens ist. Für Letzteres sage ich hier mit einem hässlichen, aber in der Forschung gängigen Wort Sprachverarbeiter (,language processor'); das Sprachlernvermögen ist nichts als die Anwendung des Sprachverarbeiters auf neues Material. Zweitens muss der Lerner Zugang zu diesem neuen Material, also zu Äußerungen der zu lernenden Sprache haben; diesen Input kann er auf unterschiedliche Weise erlangen, und die unterschiedliche Form des Zugangs spielt für Verlauf und Endresultat des Erwerbsprozesses eine wesentliche Rolle. Drittens muss es einen besonderen Grund, ein Motiv, einen Antrieb geben, den Sprachverarbeiter auf den ihm zugänglichen Input anzuwenden; dieser Antrieb ist bei Erstspracherwerb, Zweitspracherwerb und Fremdspracherwerb sehr unterschiedlich, und auch dies hat Konsequenzen für den Erwerbsprozess.

\subsection{Der Sprachverarbeiter}

Wir alle werden mit der Fähigkeit geboren, eine Sprache zu lernen und $\mathrm{zu}$ gebrauchen. $\mathrm{Ob}$ diese Fähigkeit bereits bei der Geburt voll ausgebildet ist, ist offen: das menschliche Hirn erfährt in den ersten zwei bis vier Lebensjahren zahlreiche massive Veränderungen, und es mag sein, dass diese Veränderungen auch den Sprachverarbeiter, oder zumindest Teile davon, betreffen. Man muss daher das speziesspezifische angeborene Sprachvermögen scharf trennen vom Lernvermögen $\mathrm{zu}$ einem bestimmten Zeitpunkt, sei es in der Kindheit, im Jugendalter oder als Erwachsener. Wie der „Sprachverarbeiter" eines Menschen in einem bestimmten Alter funktioniert, hängt von zweierlei ab, nämlich (a) von gewissen biologischen Determinanten, und (b) von dem gesamten Wissen, über das der Lerner zu dieser Zeit verfügt.

\subsubsection{Biologische Determinanten}

Hierzu zählen zum einen einige periphere Organe, insbesondere der Artikulationsapparat vom Kehlkopf bis zu den Lippen und der gesamte Gehörtrakt (bzw., bei geschriebener Sprache, das Sehvermögen). Zum andern hat man einige Teile der zentralen Verarbeitung im Gehirn hierhin zu stellen, also höhere Aspekte der Wahrnehmung, Gedächtnis, 
Kognition, vielleicht auch - wie von manchen Sprachtheoretikern angenommen - ein eigenes „Sprachmodul“, d. h. ein ausschließlich für die Sprache verantwortliches Teil in der Kortex. Hierfür gibt es allerdings bislang wenig empirische Belege, und es ist sicherlich ökonomischer, wenn man ohne eine solche Annahme auszukommen versucht.

Beide Arten biologischer Determinanten sind dem Menschen angeboren; beide verändern sich im Laufe des Lebens in gewissen Grenzen, und solche Veränderungen sind sicher von großer Bedeutung für den unterschiedlichen Verlauf des Spracherwerbs in verschiedenen Lebensaltern. Möglicherweise erklären sie sogar den oben erwähnten zentralen Umstand, dass der Erwerb einer zweiten Sprache im Erwachsenenalter selten zu perfekter Beherrschung führt. Viele naturgegebene biologische Prozesse, von der Prägung der Graugänse auf ihre „Mutter" bis auf die Fähigkeit, ein Kind zu gebären, sind auf eine bestimmte Spanne, eine „kritische Periode", beschränkt. Der Biologe Eric Lenneberg (1967) hat auch für den Spracherwerb eine solche kritische Periode postuliert, die etwa bis zur Pubertät reicht; danach kann auf Grund von Veränderungen in dem Kortex zwar immer noch eine Sprache gelernt werden, aber nur in jenem Sinne, in dem man sich auch Kenntnisse über die Algebra oder die großen Schlachten der Geschichte aneignet. Diese in der Erwerbsforschung oft unbesehen geglaubte Theorie hat den bestechenden Charme einfacher Erklärungen, freilich auch den Mangel, dass alle bekannten Veränderungen im Hirn des Kindes wesentlich früher liegen - etwa bis zum Abschluss des vierten Lebensjahres; Kinder im Alter von acht oder zehn Jahren haben aber in der Regel keinerlei Probleme, eine weitere Sprache perfekt zu lernen. Dies spricht nicht gegen die Relevanz von biologischen Veränderungen für das Erlernen einer Sprache, wohl aber gegen die einfache Vorstellung einer ,kritischen Periode" (für eine Würdigung und einen Überblick über die verschiedenen Alterseffekte siehe Singleton 1989).

\subsubsection{Verfügbares Wissen}

Die biologischen Determinanten setzen gleichsam den Rahmen, innerhalb dessen sich der Spracherwerb vollziehen kann. Dieser Prozess ist aber nicht momentan, er dauert lange Zeit - in der Regel viele Jahre -, und in dieser Zeit verändert sich fortwährend das dem Lerner jeweils zu Verfügung stehende
Wissen. $\mathrm{Zu}$ diesem Wissen zählt zum ersten das gesamte Welt- und Faktenwissen des Lerners zum jeweiligen Zeitpunkt. Dieses Wissen erlaubt ihm überhaupt erst, bestimmte Elemente des Schallstroms, der ihm aus seiner sozialen Umgebung entgegentönt, in kleinere Segmente aufzubrechen und diese mit einer Deutung $\mathrm{zu}$ versehen. Zum zweiten zählt dazu seine mehr oder minder begrenzte Kenntnis der zu lernenden Sprache (der Zielsprache) zum gegebenen Zeitpunkt. Der Spracherwerb ist immer ein kumulativer Prozess, bei dem Wissen auf Grund bereits vorhandenen Wissens aufgebaut wird. Drittens schließlich zählt beim gesteuerten wie beim ungesteuerten Zweitspracherwerb, nicht aber beim Erstspracherwerb, auch die Kenntnis der Erstsprache (oder auch mehrerer bereits beherrschter Sprachen) zum jeweils vorhandenen Wissen.

Dieser letzte Umstand ist die Ursache für eines der wichtigsten Konzepte des Zweitspracherwerbs - den Transfer. Wenn man bereits eine Sprache kennt, so werden die Eigentümlichkeiten der neuen Sprache im Lichte der bereits vorhandenen Sprachkenntnisse wahrgenommen und interpretiert. Für das Verständnis des Transfer ist wesentlich, dass es sich nicht etwa um eine Beziehung zwischen zwei „Sprachsystemen“ handelt, sondern um eine Interferenz zwischen verschiedenen Wissenskomponenten des Lerners $\mathrm{zu}$ einem gegebenen Zeitpunkt: das, was er $\mathrm{zu}$ dieser Zeit von der Ausgangssprache weiss, wirkt sich auf das aus, was er zu dieser Zeit von der Zielsprache weiss oder vielmehr zu wissen glaubt. Deshalb ist auch die sogenannte „kontrastive Linguistik“ nur von begrenztem Nutzen für eine Erklärung des Transfers, denn sie vergleicht im allgemeinen sprachliche Systeme oder Teile davon, nicht aber das jeweils verfügbare Wissen eines Lernenden (vgl. Art. 16; 18; 19).

Wie in allen Fällen, in denen vorhandenes Wissen den Aufbau neuen Wissens beeinflusst, in der Wissenschaft wie im Leben, kann dieser Einfluss positiv oder negativ sein; dementsprechend spricht man von positivem wie von negativem Transfer. Dabei ist sehr wohl möglich, dass ein Transfer, der ursprünglich das Lernen erleichtert hat, auf die Dauer negative Wirkungen zeitigt. Um es an einem Punkt zu illustrieren: Die Art und Weise, wie die Erstsprache bestimmte inhaltliche Bereiche, etwa den Ausdruck des Raumes und der Zeit, strukturiert, wird, sofern gewisse Ähnlichkeiten vorliegen, auf die 
Zweitsprache übertragen; dies macht das Lernen zunächst leichter, hat aber unter Umständen zur Folge, dass die feineren Unterschiede nicht bemerkt werden. Ein Deutscher, der Englisch lernt, hat zunächst weniger Schwierigkeiten mit dem englischen present-perfect als ein Chinese: he has sung und er hat gesungen sind sehr ähnlich; im Chinesischen gibt es keine vergleichbare Markierung der Temporalität durch eine periphrastische Konstruktion. Auf der anderen Seite ist eben diese Ähnlichkeit zugleich ein Grund dafür, daß die tatsächlich bestehenden subtilen Unterschiede nicht wahrgenommen werden; deshalb wird der Lerner durch den zunächst einmal positiven Transfer verführt, diese Unterschiede vielleicht nie zu lernen - jedenfalls wenn er nicht ausdrücklich darauf aufmerksam gemacht wird. Diesen gleichzeitig erleichternden und hemmenden Einfluss der Erstsprachkenntnisse finden wir auch in anderen Bereichen, beispielsweise in der Phonologie. So zeigt sich, daß spanischsprachige Arbeiter, die in der alltäglichen Kommunikation Deutsch lernen, zunächst wesentlich mehr Schwierigkeiten mit dem Konsonantismus, insbesondere mit den Konsonantenverbindungen, haben als mit dem Vokalismus; dies erklärt sich leicht durch den Einfluss der Erstsprache. Nach einer Weile kehrt sich dieses Verhältnis jedoch um - Konsonanten und Konsonantenverbindungen werden beherrscht, der Vokalismus bleibt stehen, eben wegen der relativen Ähnlichkeit (Tropf 1983). Dies erklärt zumindest teilweise eine der auffälligsten Eigenschaften des Zweitspracherwerbs - die Fossilierung. Der Lerner macht in bestimmten Bereichen keinerlei Fortschritte mehr, er ist nicht mehr aufnahmebereit für neuen Input bestimmter Art, obwohl seine Sprache von der seiner sozialen Umgebung noch weit entfernt ist. Es kann dies aber nicht die einzige Erklärung dafür sein, dass nur der Erstspracherwerb in der Regel zu perfekter Beherrschung führt: Im Fremdspracherwerb wird der Lerner nämlich ausdrücklich auf diese Unterschiede hingewiesen, und dennoch ist er oft nicht in der Lage, sie sich zu eigen zu machen (Überblicke über den gegenwärtigen Stand der Transferforschung geben Kellerman und Sharwood-Smith 1986; Odlin 1989; Ellis 1994, 299-346).

\subsection{Der Input}

Der Sprachverarbeiter ist bei Geburt bei allen Menschen gleich - im Rahmen der Schwankungsbreite aller angeborenen Eigen- schaften. Es ist dem einzelnen nicht angeboren, Mopan oder Twi zu lernen. Dass sich der Lerner eine bestimmte Sprache mit all ihren strukturellen Besonderheiten aneignet, liegt daran, dass die soziale Umwelt des Lerners diese Sprache spricht und sie als Input für seinen Sprachverarbeiter zugänglich macht.

Wie wird dem Lerner die Sprache der sozialen Umgebung zugänglich? Er versteht diese Sprache ja noch nicht, er hat, mit dem heiligen Thomas von Aquin zu reden, von der Rede nur den flatus vocis, aus dem er einen gewissen Sinn zu machen versuchen muss. Er sieht sich in der Lage eines Menschen, der sich plötzlich in eine Welt versetzt findet, in der alle um ihn herum nur Malayalam reden. Anders gesagt: Der Input besteht zunächst einmal aus unanalysierten Schallwellen, die das Ohr des Lerners treffen, also nichts, was bereits in Phoneme, Wörter, Sätze zerlegt wäre. Den Schallstrom in kleinere Einheiten aufzubrechen und diese mit einem bestimmten Sinn zu versehen, ist die erste Aufgabe des Lerners, und wenn er dazu nur die Schallwellen zur Verfügung hätte, dann wäre sie nicht zu lösen. Wenn man einen Lerner Tage, Wochen, ja Jahre in ein Zimmer einsperren und mit Inuktitut beschallen würde, so würde er es doch nicht lernen. Man benötigt dazu auch die gesamte begleitende Information, Gesten, Handlungen, den ganzen situativen Kontext, mit dessen Hilfe es möglich ist, einzelne Teile aus dem Schallstrom herauszubrechen und sinnvoll zu interpretieren. Mit anderen Worten, der Input besteht eigentlich aus zwei parallelen Informationsquellen - dem Schallstrom und der gesamten situativen Parallelinformation. An diesen kann der Sprachverarbeiter ansetzen, um das Analyseproblem des Spracherwerbs zu lösen. Dabei spielt nun das gesamte jeweils verfügbare Wissen eine wesentliche Rolle, insbesondere Kenntnis der strukturellen Regularitäten einer bereits vorhandenen Sprache, die den Lerner bei der Lösung des Analyseproblems leiten und eben auch fehlleiten können. Dies gilt in gleicher Weise für den Erstspracherwerb wie für den ungesteuerten Zweitspracherwerb: Säugling wie Erwachsener hören zunächst nur blahblahblah in einem sozialen Kontext, und sie müssen ersteres mit Hilfe des letzteren deuten. Wie dies im Einzelnen geschieht, was also die natürlichen Gesetzlichkeiten des menschlichen Spracherwerbs sind, lässt sich nur empirisch klären.

Beim gesteuerten Zweitspracherwerb ist dies in einem wesentlichen Punkt anders: die 
zu lernende Sprache wird dem Sprachverarbeiter zu großen Teilen nicht direkt zugänglich gemacht, sondern in Form einer linguistischen Beschreibung. Es wird gesagt, die strukturellen Gesetzlichkeiten einer Sprache sind so und so, eigne sie dir an! Das Sprachlernvermögen muss sich nicht dem Material selbst, sondern mit einer bestimmten Aufbereitung dieses Materials auseinandersetzen. Darauf ist der menschliche Sprachverarbeiter, so wie er sich im Laufe der Jahrtausende, vielleicht Jahrhunderttausende entwikkelt hat, nicht zugeschnitten. Das besagt keineswegs, dass das Ergebnis des Fremdspracherwerbs ein schlechteres sein müßte (in der Praxis ist oft das Gegenteil der Fall). Es besagt aber wohl, dass die Gesetzlichkeiten, die den Prozess des Fremdspracherwerbs kennzeichnen, teilweise andere sind als beim Zweitspracherwerb: Sie resultieren aus dem Versuch, in einen natürlichen, durch bestimmte Charakteristika gekennzeichneten Prozess von außen her steuernd einzugreifen. Die Folge ist, dass der gesteuerte Zweitspracherwerb durch die Interaktion dreier Formen sprachlichen Wissens gekennzeichnet ist. Dies sind:

(a) Das intuitive Wissen über die strukturellen Eigenschaften der Erstsprache (oder auch mehrerer bereits beherrschter Sprachen).

(b) Das ,intuitive“, d.h. durch die üblichen Prozesse des Spracherwerbs zustandegekommene partielle Wissen über die Zweitsprache; selbst bei ausgeprägt grammatischem Unterricht muss sich der Lerner ja auch mit dem Material selbst (hier oft in seiner geschriebenen Form) auseinandersetzen. Dieses Wissen kann man das (intuitive) Eigenwissen des Lerners nennen - das, was er selbst intuitiv aus dem ihm zugänglichen Input abgeleitet hat.

(c) das Beschreibungswissen über die Zweitsprache, d.h. jene partielle Kenntnis der Zweitsprache, die auf Grund metalinguistischer Beschreibung dieser Zweitsprache zustandegekommen ist und das dem Lernenden durch den Unterricht oder durch Lehrbücher nahegebracht worden ist.

Sprechen und Verstehen eines FremdsprachLerners zu einem gegebenen Zeitpunkt werden daher nicht von einem einheitlichen, zugrundeliegenden Wissen bestimmt, sondern von einer eigentümlichen Mischform mit drei Anteilen in variierender Gewichtung. Wie diese zusammenwirken, ist unklar. Auf Krashen geht die Vorstellung zurück, dass be- wusst gelerntes Wissen, sofern genügend Zeit vorhanden ist, das unbewusst erworbene Wissen wie eine Überwachungsinstanz, ein „Monitor" steuert (zusammenfassend dargestellt in Krashen 1981). Dies ist aber eher eine schöne Metapher, die suggestiv beschreibt, wie man in einem gegebenen Fall bei der Produktion von Äußerungen in der Zweitsprache vorgeht; aber sie sagt wenig über die Gesetzlichkeiten, nach denen sich der Erwerbsprozess entfaltet. Die „Monitortheorie“ hat in der Sprachlehrforschung einen ganz erstaunlichen Widerhall gefunden, allerdings mehr im Sinne eines deklamatorischen Überbaus; für die konkrete Anwendung oder gar für die empirische Erforschung des Spracherwerbs ist sie zu wenig konkret, so dass sie hier kaum eine Rolle gespielt hat.

Es ist eine interessante und bislang wenig studierte Frage, was passiert, wenn Beschreibungswissen - also das, was man dem Lerner im Unterricht als Regel vorgegeben hat - und induktiv abgeleitetes Eigenwissen über die Zweitsprache in Widerstreit geraten. Eine der wenigen Untersuchungen zu dieser Frage, Carroll u. a. (1982) zeigt, dass dieser Konflikt bei Sprechern unterschiedlicher Herkunft, in diesem Falle Amerikanern und Japanern, unterschiedlich aufgelöst werden kann: letztere gewichten das Beschreibungswissen wesentlich höher als erstere, ein Umstand, der sich im Korrekturverhalten deutlich niederschlägt. Sie sind, um so etwas anders zu formulieren, Interferenzen aus dem Beschreibungswissen eher zugänglich als die Amerikaner, weil sie offenbar eine andere Einstellung dazu haben, was eigentlich gelernt werden soll. Dies bringt uns auf die letzte Komponente, die bei jedem Spracherwerb unabdinglich ist - den spezifischen Antrieb, der den Lerner veranlasst, seinen Sprachverarbeiter auf einen bestimmten Input anzuwenden.

\subsection{Antrieb}

Es gibt verschiedene, zumeist Hand in Hand gehende Gründe, aus denen man eine Sprache lernen kann. Der wichtigste ist ohne Zweifel die soziale Integration. Ein Kind muss eine bestimmte soziale Identität erwerben, und dazu muss es die Sprache seiner sozialen Umgebung exakt reproduzieren. Es muss sich daher im sprachlichen (wie im sonstigen) Verhalten auch bestimmte Gewohnheiten zu eigen machen, deren Sinn es nicht sieht, weil sie keinen haben - im Deutschen beispielsweise die Genusunterscheidung, den Unterschied zwischen starken und schwachen 
Verben oder eine bestimmte Ausspracheweise, etwa ein stimmhaftes /s/ im Silbenanlaut. Ein Kind, das das /s/ in allen Positionen gleich ausspräche, konsequent bei allen $\mathrm{Ob}$ jekten den Artikel "das" verwendete und „laufte“ und „schwimmte“ statt „lief" und „schwamm" sagen würde, wäre im Grunde viel logischer und vernünftiger als seine soziale Umgebung - aber es wäre in dieser Umwelt ein Außenseiter. Es tut daher gut daran, alle Ungereimtheiten, sprachliche und sonstige, im Verhalten der sozialen Umgebung getreulich mitzumachen. Diese Notwendigkeit entfällt bei dem zweiten wesentlichen Grund, eine Sprache zu lernen - den kommunikativen Bedürfnissen. Man kann keine soziale Integration anstreben, ohne auch bestimmte kommunikative Bedürfnisse verwirklichen $\mathrm{zu}$ wollen; deshalb schließt der erste Grund den zweiten in einer bestimmten Ausprägung ein; aber umgekehrt will nicht jeder, der spanische Handelskorrespondenz lernt, auch ein Spanier werden. Die kommunikativen Bedürfnisse können sehr unterschiedlich sein, und dies wirkt sich in aller Regel auf Verlauf und Endzustand des Erwerbsprozesses aus. Ein ausländischer Arbeiter, der für einige Zeit nach Deutschland kommt, will in bestimmten Grenzen andere verstehen und sich selbst verständlich machen können; aber er muss nicht den Eindruck erwecken wollen, er wäre ein Hiesiger. Wenn ihm dies auch noch gelingt, so mag das mancherlei Vorteile haben; aber es ist nicht einfach zu erreichen, und es ist vielleicht gar bedrohlich für die eigene soziale Identität.

Soziale Integration und kommunikative Bedürfnisse sind sicher die wichtigsten natürlichen Antriebsfaktoren im Spracherwerb. Sie spielen hingegen im schulischen Fremdsprachenerwerb zumeist eine untergeordnete Rolle. Latein lernt man, weil es zu einer bestimmten Vorstellung von Bildung gehört, und selbst der Englischunterricht auf dem Gymnasium ist nicht vornehmlich durch die kommunikativen Bedürfnisse der Schüler motiviert. Auf diesen Antriebsfaktor, sich eine Sprache anzueignen, ist das menschliche Sprachvermögen von Natur aus nicht angelegt, und deshalb bedarf es in diesem Falle gewöhnlich zusätzlicher Motivationen; dazu zählt beispielsweise der Druck der Noten, aber auch eine bestimmte emotionale Einstellung zum Gegenstand, die der Lehrer mit Glück und Geschick erzeugen kann. Die positive, manchmal auch negative Wirkung solcher affektiver Faktoren im Spracherwerb ist hinlänglich bekannt; woraus sie diese Wirkung auf die Sprachverarbeitung - also einen psychischen Prozess, der sich irgendwo in unserem Hirn abspielt - beziehen, ist allerdings gänzlich ungeklärt (vgl. neuerdings jedoch Pulvermüller und Schumann 1994).

\section{Der Prozess des Spracherwerbs}

Keine unter den vielen Formen des Spracherwerbs ist abrupt: es handelt sich stets um einen langwierigen, graduellen Prozess, der bestimmten, in Grenzen variablen Gesetzlichkeiten folgt. Dies ist offenkundig; aber es wird in der Forschung nicht immer ernst genommen. In einer der einflussreichsten Richtungen der Spracherwerbsforschung, der nativistischen Theorie der generativen Grammatik, wird sogar ausdrücklich vom Entwicklungscharakter abstrahiert - nicht weil man ihn bestreiten würde, sondern weil, so die Annahme, er für das Verständnis des Erwerbs nicht wesentlich ist. Aber auch andere Betrachtungsweisen versuchen in der Praxis selten, die innere Logik der Entwicklung aufzuklären; wir kommen darauf in $\mathrm{Ab}$ schnitt 4. zurück.

Wie jeder Prozess ist auch dieser durch drei Faktoren gekennzeichnet: Anfangszustand, Endzustand und Verlauf. Bei letzteren ist es sinnvoll, zwischen Tempo und Struktur zu unterscheiden. Mit Struktur des Verlaufs ist gemeint, in welcher Abfolge welche strukturellen Eigenschaften aus dem Input herausgearbeitet werden, mit Tempo des Verlaufs die unter Umständen wechselnde Geschwindigkeit, mit der dies geschieht. Der Anfangszustand ist einfach das Wissen, über das der Lerner zu Beginn des Lernprozesses verfügt (siehe oben Abschnitt 2.1.2.), und das ist, was die spezifischen Eigenschaften der Zielsprache angeht, zunächst einmal null. Dennoch mag der Lerner auch zu Beginn schon einiges über die Zielsprache wissen - nämlich all jenes, was allen Sprachen gemeinsam ist („Universalien“) sowie all das, was Zielsprache und bereits beherrschte Sprachen gemeinsam haben. Über das Tempo des Verlaufs ist außer anekdotischen Beobachtungen wenig bekannt. Wir gehen deshalb im Folgenden nur auf Struktur des Verlaufs und Endzustand ein.

\subsection{Struktur des Verlaufs}

Bei allen Formen des ungesteuerten Erwerbs, also beim Erstspracherwerb wie beim Zweit- 
spracherwerb im sozialen Kontext, ergibt sich die Struktur des Verlaufs daraus, wie es dem Sprachverarbeiter gelingt, den Input in elementare Einheiten aufzubrechen, diese in ihrer Funktion zu deuten und die Regeln $\mathrm{zu}$ entschlüsseln, nach denen sie sich zu komplexeren Einheiten verbinden lassen. Alles, was dem Lerner dazu zur Verfügung steht, ist seine biologisch gegebene Lernfähigkeit, sein Ausgangswissen sowie die beiden Komponenten des Inputs - der Schallstrom und die parallele situative Information. Gehen alle Lerner diese Aufgabe in gleicher Weise an? Das ist, wie schon die Alltagserfahrung zeigt, offenkundig nicht der Fall; es gibt eine gewisse Variabilität im ungesteuerten Spracherwerb. Die Frage ist bloß, wie weit sie geht und wovon sie abhängt. Beliebig kann sie jedenfalls nicht sein. Dies ergibt sich allein schon daraus, dass der Erwerb mancher Eigenschaften das Vorhandensein anderer voraussetzt. In den meisten Sprachen hängt die Grundwortstellung im Satz vor allem von der Position der Finitheitsmarkierung, und damit des finiten Verbs, ab. Im Deutschen steht die finite Komponente des Verbs im Hauptsatz normalerweise an zweiter Stelle, die infinite Komponente an letzter (Heute HAT er eine Rede gehalten). Wenn finite und infinite Komponente zu einer morphologischen Form verschmolzen sind, dann ist die Stellung der finiten Komponente maßgeblich (Heute hält er eine Rede). Um diese (hier vereinfacht dargestellte) Grundregel der deutschen Syntax aus dem Input abzuleiten, muss der Lerner wissen, wie die Finitheit im Deutschen markiert wird. Dies geschieht durch eine bestimmte Art der Verbflexion (hat, hält), also durch eine morphologische Markierung. Diese wiederum kann er erst erkennen, wenn er zumindest bestimmte Grundzüge der deutschen Phonologie gelernt hat, beispielsweise dass /a:/ und /æ:/ phonologisch distinkt sind. Solche und ähnliche Abhängigkeiten schränken die Struktur des Verlaufs ein. Aber sie lassen immer noch ein erhebliches Spektrum möglicher Entwicklungen zu, über die es nach wie vor mehr Spekulationen als empirisch gut gesicherte Erkenntnisse gibt.

Im durch Unterricht gesteuerten Spracherwerb hängt der Verlauf des Prozesses nun außerdem davon $a b$, in welcher Weise die strukturellen Eigenschaften der Zielsprache präsentiert werden - vom "Curriculum“. Dieser Faktor setzt die im ungesteuerten Erwerb wirksamen Lernprozesse sicher nicht außer Kraft; aber es ist unklar, welches Ge- wicht ihm zukommt - ob, etwas zugespitzt formuliert, die Lerner wegen oder trotz des Unterrichts Fortschritte machen. Allgemeine Aussagen sind schon deshalb problematisch, weil Art und relatives Gewicht dessen, was dem Lerner in Form von Beschreibungswissen vermittelt wird, je nach Art des Unterrichts erheblich schwanken. Sie verbieten sich aber auch deshalb, weil wir die Gesetzlichkeiten des ungesteuerten Spracherwerbs, in die der Unterricht optimierend $\mathrm{zu}$ intervenieren sucht, allenfalls in Ansätzen kennen.

\subsection{Endzustand}

Der Spracherwerb ist abgeschlossen, wenn der Lerner die Gesetzlichkeiten der Zielsprache beherrscht. Dies ist beim Erstspracherwerb wie beim ungesteuerten Zweitspracherwerb die Sprache der sozialen Umgebung, die den Input liefert; bei gesteuertem Spracherwerb ist es eine normative Beschreibung, die die Lehrenden für besonders wünschenswert halten („King's English“, das Französische, so wie es in Grevisse und Petit Robert beschrieben ist, usw.). Beim Erstspracherwerb wird das Ziel gewöhnlich erreicht; jede Abweichung davon gilt als pathologisch. Beim Zweitspracherwerb im Erwachsenenalter wird es hingegen in aller Regel verfehlt: der Erwerbsprozess kommt schon früher zum Stillstand, er fossiliert auf irgendeiner Stufe, die der Zielsprache mehr oder minder nahekommt. Wie erklärt sich dieser Unterschied? Art und Menge des Inputs können nicht, oder jedenfalls nicht allein, verantwortlich sein, denn Kinder und ungesteuert lernende Erwachsene haben im Wesentlichen denselben Input. Umgekehrt ist der Input bei gesteuertem und bei ungesteuertem Zweitspracherwerb sehr verschieden, in beiden Fällen aber ist Fossilierung der Normalfall. Die Fossilierung korreliert jedoch offensichtlich mit dem Alter. Das deutet darauf, dass altersbedingte Veränderungen im Sprachverarbeiter verantwortlich sind. Dies können Veränderungen in den biologischen Gegebenheiten (den beteiligten Teilen der Hirnrinde oder in den peripheren Organen) sein, oder aber Unterschiede im jeweils verfügbaren Wissen, und hier ist der wesentliche Faktor das Vorhandensein einer oder mehrerer anderer Sprachen. Beide Möglichkeiten sind sehr plausibel, und wir haben sie oben unter den Stichwörtern „kritische Periode“ und „(negativer) Transfer" bereits angesprochen. Beide aber können nicht erklären, weshalb Kinder vor der Pubertät, etwa im Alter von acht Jahren, 
gewöhnlich keine Fossilierung zeigen: sie haben kein Problem, eine zweite oder dritte Sprache ,perfekt' zu lernen (McLaughlin 1978). Die Kinder ausländischer Arbeiter sind ein bekanntes Beispiel. Möglicherweise liegt der Hauptgrund für die Fossilierung daher in der unterschiedlichen Form des Antriebs, insbesondere zwischen dem unbewussten Wunsch nach sozialer Integration, der perfekte Nachahmung verlangt, und anderen Motivationen, etwa dem Wunsch, sich zu begrenzten Zwecken verständlich zu machen oder aber das Abitur zu bestehen. Dies schließt nicht aus, dass auch andere Faktoren wirksam sind; schließlich lässt das Lernvermögen mit zunehmendem Alter nicht nur in Sachen Spracherwerb nach; aber das Gewicht verlagert sich etwas von biologischen auf sozialpsychologische Faktoren (Klein 1995).

\section{Die wesentlichen Theorien}

Wenn sich das Interesse der Forschung einem neuen Gebiet zuwendet, so lässt es sich gewöhnlich von Methoden, Leitfragen und theoretischen Überlegungen leiten, von denen die Forscher meinen, dass sie sich in anderen, verwandten Gebieten bewährt haben. Die ernsthafte Erstspracherwerbsforschung ist gut hundert Jahre alt, und sie ist aus der Psychologie entstanden: Forscher wie Carl Preyer, Wilhelm Wundt, Clara und William Stern haben sich dafür interessiert, welche Rolle die Sprache in der gesamten kognitiven und sozialen Entwicklung des Kindes spielt, und so ist denn in dieser Tradition die Spracherwerbsforschung treu und brav und mit einiger Verzögerung den Mäandern der psychologischen Theoriebildung gefolgt. Ein zweiter, diesmal von der strukturellen Linguistik inspirierter Traditionsstrang setzt vor sechzig Jahren mit Roman Jakobson ein; prägend für diese linguistische Linie war vor allem Chomskys Vorstellung, wesentliche Elemente der Grammatik seien angeboren (und folglich universal), weil nur so der leichte, schnelle und von den Unzulänglichkeiten des Inputs unabhängige Spracherwerb des Kindes zu erklären sei. Diese „,nativistische Theorie" hat verschiedene Wandlungen erfahren; für ihre heutige Form ist vor allem der Gedanke des ,parameter setting“ bestimmend; wir kommen gleich darauf zurück (einen Überblick über die verschiedenen Theorien des Erstspracherwerbs geben Slobin 1985, Bd. 2, sowie Fletcher und MacWhinney 1995).
Die Suche nach den Gesetzlichkeiten des Zweitspracherwerbs ist demgegenüber relativ jung; sie setzt vor etwa dreißig Jahren ein und hat drei wesentliche Inspirationsquellen; dies ist zum Einen der Fremdsprachunterricht und die Beobachtungen, die dabei über die Lernschwierigkeiten der Schüler gemacht worden sind, zum Zweiten die Forschung zum Erstspracherwerb, und zum Dritten jener Teil der Linguistik, der sich empirisch und theoretisch mit Problemen der sprachlichen Variabilität befasst - Dialektologie, Pidginforschung, Soziolinguistik. Anfänglich über diese Quellen vermittelt, dann auch direkt gehen in die Zweitspracherwerbsforschung seither wiederum die jeweils modischen Vorstellungen aus der Psychologie, der theoretischen Linguistik und der Psycholinguistik ein.

Es gibt derzeit keine Theorie, die auch nur annähernd in der Lage wäre, den verschiedenen Formen des Spracherwerbs gerecht zu werden. Dies liegt an der Fülle der Faktoren, die darin eine Rolle spielen, und an der Schwierigkeit, einen so langwierigen und variablen Prozess empirisch zu untersuchen; das in den Abschnitten 2. und 3. Gesagte sollte dies deutlich gemacht haben. Dennoch haben sich kühne Forscher und Forscherinnen nicht abhalten lassen, allgemeine Theorien des Spracherwerbs aufzustellen. Ihre Reichweite ist gewöhnlich sehr begrenzt, sowohl was die Zahl ihrer Anhänger angeht wie nach dem Gegenstandsbereich, den sie tatsächlich abdecken. Im Folgenden konzentrieren wir uns, den Zielen dieses Handbuchs entsprechend, auf den Erwerb einer zweiten Sprache und diskutieren kurz zwei solcher Theorien, die in der Vergangenheit eine wesentliche Rolle gespielt haben, und zwei weitere, die das Bild der Forschung heute bestimmen (eine umfassende Darstellung findet sich in Ellis 1994 sowie, freilich stark aus amerikanischer Perspektive, in Ritchie und Bathia 1996).

\subsection{Identitätshypothese}

Am einfachsten wäre es, wenn alle Arten des Spracherwerbs denselben Gesetzlichkeiten folgen würden. Diese Auffassung ist in der Tat vertreten worden, allerdings stets bezogen auf einige Einzelfälle, etwa die Reihenfolge, in der bestimmte Flexionsmorpheme im Englischen gelernt werden, oder den Erwerb der Negation (siehe dazu zusammenfassend Wode 1981). Insgesamt sind jedoch die Unterschiede zwischen den einzelnen Erwerbsformen zu offensichtlich, als dass man 
eine solche Beobachtung verallgemeinern könnte. Manche Formen zeichnen sich typischerweise durch Fossilierung aus, andere nicht, und zumal im gesteuerten Zweitspracherwerb hängt die Struktur des Verlaufs entscheidend von der Präsentation des Materials ab; wenn das simple past nach dem past progressive gelehrt wird, kann jenes nicht zuerst auftauchen. All dies schließt freilich nicht aus, dass es allgemeine Prinzipien des Spracherwerbs gibt; diese müssen aber auf einer relativ abstrakten Ebene liegen, wie dies beispielsweise in der Theorie des ,parameter setting" angenommen wird. (vgl. Abschnitt 4.3.); nur ausnahmsweise äußern sie sich tatsächlich in identischem Verlauf, identischem Tempo und identischem Endzustand.

\subsection{Kontrastivitätshypothese}

Beim gesteuerten wie beim ungesteuerten Zweitspracherwerb muss man sich lediglich jene strukturellen Eigenschaften aneignen, in denen sich die zweite Sprache von der ersten unterscheidet. Daraus kann man ableiten, dass ein Ausdrucksmittel, sei es ein Wort oder eine Konstruktion, um so schwieriger zu lernen ist, je stärker die beiden beteiligten Sprachen sich darin unterscheiden; möglicherweise bestimmt der Grad der Kontrastivität sogar Tempo und Struktur des Erwerbsprozesses. Dieser Gedanke hat zumindest eine erhebliche Anfangsplausibilität, und so ist er denn seit den Fünfzigerjahren immer wieder in verschiedenen Varianten vorgetragen worden. Nicht zuletzt hat er den Anstoß zu einer Reihe von „kontrastiven Grammatiken“ gegeben. Ihr Nutzen für die Spracherwerbsforschung ist allerdings sehr begrenzt. Das hat eine ganze Reihe von Gründen. Zum Ersten kommt es für den Spracherwerb nicht darauf an, wo und wie sich zwei sprachliche Systeme unter irgendeiner linguistischen Analyse unterscheiden, sondern darauf, was sich davon im Wissen des Lerners zu einem gegebenen Zeitpunkt widerspiegelt: der Lerner muss nicht zwei Systeme zueinander in Bezug setzen, sondern seine Repräsentation der Ausgangssprache zu seiner Deutung des Inputs. Zum Zweiten ist ein massiver Unterschied gewöhnlich viel leichter zu erkennen als ein subtiler, und demnach sollte er auch leichter zu lernen sein. Zum Dritten zählen zur Sprachbeherrschung sowohl Verstehen wie Produktion, und eine strukturelle Eigenschaft mag leicht zu verstehen, aber schwer $\mathrm{zu}$ produzieren sein, und umgekehrt. Zum Vierten hängt die Struktur des Erwerbs of- fenkundig von vielen anderen Faktoren ab, etwa der Häufigkeit im Input, der kommunikativen Relevanz - ein türkischer Arbeiter lernt nicht jene Wörter oder Konstruktionen zuerst, die dem Türkischen möglichst ähnlich sind, sondern jene, die er besonders dringend benötigt - oder auch den in Abschnitt 3.1. beschriebenen Abhängigkeiten zwischen strukturellen Eigenschaften. All dies besagt nicht, dass kontrastive Aspekte keinerlei Rolle im Zweitspracherwerb spielen; aber sie sind nur ein Faktor unter vielen, und wahrscheinlich kein besonders wichtiger.

\subsection{Universalgrammatik und „,parameter setting"}

In der Geschichte der Sprachwissenschaft, sowohl der traditionellen wie der strukturellen, ist der Spracherwerb eine Randerscheinung. Ein hoher theoretischer Status kommt ihm jedoch in der Generativen Grammatik Chomskys und seiner Schüler zu. Jedes Kind, so eine Annahme der generativen Grammatik, lernt normalerweise seine Muttersprache auch bei fehlerbehaftetem und unzulänglichem Input schnell und mühelos bis zur „Perfektion"“. Dies ist nicht durch einen induktiven Lernprozess zu erklären; vielmehr muss ein wesentlicher Teil der Sprachbeherrschung angeboren sein und lediglich in den ersten Lebensjahren aktiviert werden. Da aber keinem eine bestimmte Sprache angeboren ist, ist der Kern der menschlichen Sprachfähigkeit universal. Der eigentliche Gegenstand der linguistischen Theorie ist daher die Universalgrammatik.

Diesen Gedanken hat Chomsky erstmals Mitte der Fünzigerjahre vorgetragen, und er hat sich durch alle Wandlungen der generativen Grammatik erhalten. Er hatte allerdings lange den Status eines theoretischen Arguments, ohne irgendwelche Konsequenzen für die tatsächliche Erforschung des Spracherwerbs. Erst zu Beginn der Achzigerjahre wurde er soweit konkretisiert, dass man ihn in konkrete empirische Forschungsprojekte - zunächst nur beim Kind, dann auch im Zweitspracherwerb - ummünzen konnte. Entscheidend dafür war der Begriff des „Parameters". Die Idee ist, sehr kurz gesagt, folgende (vgl. Chomsky 1981). Die gesamte Kompetenz eines erwachsenen Sprechers, der seine Sprache perfekt beherrscht, besteht aus einem "Kern" (core) und der "Peripherie“. Letzteres sind alle idiosynkratischen Erscheinungen einer Sprache, also beispielsweise die besondere deutsche Form der Nominalfle- 
xion oder die besondere Laut-BedeutungZuordnung eines einzelnen deutschen Wortes; dass das Buch im Deutschen /bu: $\mathrm{x} /$ heißt und nicht beispielsweise / kni:ga/, dass der Genitiv davon /bu:xəs/ ist und nicht/ba:x/ dies sind Idiosynkrasien des Deutschen. Der Kern ist im Prinzip angeboren und universal; jedoch lässt die Universalgrammatik zu Beginn bestimmte Alternativen offen, die dann einzelsprachlich unterschiedlich ausfallen. Ebendies sind die Parameter. Ein besonders einfaches Beispiel ist der ,head parameter": jede Konstruktion hat einen „Kopf“ und ein „Komplement"; in frisches Brot ist Brot der Kopf und frisch das Komplement; in Brot essen ist essen der Kopf und Brot das Komplement. Die Universalgrammatik lässt zunächst offen, ob das Komplement dem Kopf folgt oder ihm vorausgeht: diese Eigenschaft ist parametrisiert, sie muss einzelsprachlich unterschiedlich belegt werden. Die Attraktivität dieses Gedankens liegt nun darin, dass ein einzelner Parameter oft eine ganze Reihe von strukturellen Eigenschaften zusammenfasst. Ein gutes Beispiel - das demnach auch im Spracherwerb am meisten untersucht wurde - ist der „Pro-drop parameter": manche Sprachen, wie Deutsch, Englisch, Französisch, haben ein obligatorisches Subjektpronomen, während andere, wie Lateinisch, Italienisch, Spanisch, es „auslassen“ können. Diese Eigenschaft des „pro-drop“ korreliert nun mit einer Reihe weiterer, insbesondere einer relativ reichen Verbalmorphologie, einer vergleichsweise freien Wortstellung sowie mit der Möglichkeit, Elemente aus bestimmten Nebensätzen herauszubewegen (dem sogenannten ,that-trace effect"). Der Pro-drop parameter besagt nun, das all diese Eigenschaften davon abhängen, wie ein bestimmtes abstraktes Element der Satzstruktur („AGR“) parametrisiert ist. Man beachte, dass die Auslassbarkeit des Subjektpronomens nicht der Parameter ist - sie ist eine von verschiedenen strukturellen Konsequenzen einer bestimmten Parametrisierung. Wenn der Parameter also erst einmal gesetzt ist - und das kann der Lerner im Prinzip an Hand jeder seiner strukturellen Konsequenzen im Input lernen -, dann folgen daraus automatisch auch alle anderen strukturellen Eigenschaften, darunter auch solche, die dem Input vielleicht nur sehr schwer zu entnehmen sind.

Der Spracherwerb hat demnach zwei Komponenten: alle Eigenschaften der Peripherie müssen aus dem Input gelernt werden, und ebenso muss die jeweils gültige Parametrisierung aus dem Input abgeleitet werden. Das wirft zwei Fragen auf: Erstens, welche Eigenschaften zählen zur Peripherie, welche zum Kern, und zweitens, was innerhalb des Kerns ist in welcher Weise parametrisiert? Die Antwort auf beide Fragen nimmt der Theorie viel von ihrem theoretischen Glanz. Aus dem Input gelernt werden muss auf jeden Fall der gesamte Wortschatz, ebenso die spezifische Form der verschiedenen morphologischen Teilsysteme, etwa die Nominalflexion, ebenso das spezifische Phoneminventar, und nicht zuletzt alle Idiosynkrasien der einzelsprachlichen Syntax - mit anderen Worten, fast alles. Nicht gelernt werden muss, was universal ist, abgesehen von jenen Eigenschaften, in denen die Universalgrammatik parametrisiert ist. Was aber sind nun die einzelnen Parameter? Darüber gibt es in der generativen Grammatik keinerlei Einigkeit; die beiden oben Genannten haben in der Erwerbsforschung eine große Rolle gespielt; aber sie haben sich zum Einen empirisch nicht bestätigen lassen, und sie sind inzwischen auch in der linguistischen Theorie selbst weithin aufgegeben. Dies macht es, bei aller Attraktivität des Gedankens, sehr schwer, seinen empirischen Gehalt zu überprüfen.

Die Idee des Parametersetzens wurde zunächst nur für den Erstspracherwerb verfolgt (eine Übersicht geben Weissenborn, Goodluck und Roeper 1992), und erst mit einiger Verzögerung auf den Zweitspracherwerb übertragen (White 1989; Epstein u.a. 1997). Die Diskussion wurde dabei von zwei Fragen bestimmt: (1) In welcher Weise können und müssen Parameter „neugesetzt" werden? Beim Erstspracherwerb sind die Parameter ja zunächst offen, und das Kind muss aus dem Input ableiten, welche Parametrisierung für seine Muttersprache gilt. Wird eine weitere Sprache gelernt, so gibt es zwei Möglichkeiten: entweder die bisherige Parametrisierung wirkt weiter, und der Lerner wird in seinem Erwerb, sei es positiv, sei es negativ, davon beeinflusst, oder aber die Universalgrammatik wirkt so wie beim Kind, d. h. der Lerner geht den Input ganz neutral an. (2) Ist die Universalgrammatik nur beim Kinde wirksam, oder ist sie auch noch für den erwachsenen Zweitsprachlerner ,zugänglich“? Diese Frage ist mit der ersten verwandt; es ist aber nicht die gleiche, denn es könnte auch sein (etwa im Sinne der Lennebergschen ,kritischen Periode"), dass die Beschränkungen 
der Universalgrammatik im fortgeschrittenen Alter überhaupt nicht mehr wirksam sind; dann müsste es auch möglich sein, ,,unmögliche Sprachen" zu lernen, d. h. solche, die dem angeborenen menschlichen Sprachvermögen nicht entsprechen. Die letzte Frage ist bislang nicht empirisch überprüft worden. Zur Ersten gibt es hingegen eine ganze Reihe von Untersuchungen; sie gelten durchweg sehr fortgeschrittenen Lernern, die ihre Kenntnisse im Unterricht erworben haben, so dass man hier sicher nicht das freie Wirken der natürlichen menschlichen Sprachverarbeitung misst. Die Ergebnisse sind kontrovers. Was gezeigt werden konnte, ist das Weiterwirken bestimmter struktureller Eigenschaften der Ausgangssprache, also etwa Auslassbarkeit und Nichtauslassbarkeit des Subjektpronomens; dies ist aber auch durch einfachen Transfer zu erklären (wie man ihn in diesem Modell bei der „Peripherie“ erwartet) und belegt nicht unbedingt ein „Neusetzen“ eines ganzen Parameters, in dem ja viele solcher struktureller Erscheinungen gebündelt sind.

\subsection{Lernervarietäten}

Allen bisher diskutierten Theorien ist eigen, dass sie den Erwerbsprozess vorrangig unter dem Gesichtspunkt des verpassten Ziels betrachten: es gibt einen Standard, den der Lerner erreicht haben sollte - nämlich die Zielsprache -, und man bemisst den Erwerb daran, wo und warum er dieses Ziel (noch) verfehlt. Unter normativen Aspekten ist dies eine höchst verständliche Betrachtungsweise, und ein Lehrer ist gut beraten, sie einzunehmen. Sie hat auch den nicht zu unterschätzenden Vorzug der methodischen Einfachheit: man ermittelt einfach die „Fehler" des Lerners, d.h. seine Abweichungen vom Ziel, und versucht, ihr Zustandekommen zu erklären. Dies erklärt den erstaunlichen Erfolg der „Fehleranalyse“ (Corder 1981) bei der Erforschung des gesteuerten Zweitspracherwerbs. Es ist aber wenig wahrscheinlich, dass man auf diese Weise versteht, was im Erwerbsprozess eigentlich abläuft: dies wird davon bestimmt, wie der Lerner gewisse Fertigkeiten aus bestimmten Gründen auf einen bestimmten Input anwendet. Wenn man die immanente Gesetzlichkeit dieses Prozesses verstehen will, muss man den Lerner selbst in den Mittelpunkt rücken. Der Gedanke, dass die Äußerungen eines Lerners $\mathrm{zu}$ irgendeinem Zeitpunkt nicht nur schlechte Nachahmungen sind, sondern ihre eigene Systematik aufweisen, ist erstmals Anfang der Siebzigerjahre unter Schlagwörtern wie ,interlanguage“, „approximate systems", ,interim systems“ und ähnlichen aufgekommen. Diese zunächst noch sehr vagen Vorstellungen sind in der Folge in einer Reihe von empirischen Projekten konkretisiert worden. Heute spricht man meist von „Lernervarietäten“, und die meisten Untersuchungen zum Zweitspracherwerb außerhalb des Unterrichts folgen dieser Betrachtungsweise (Meisel u. a. 1981; Perdue 1983; Klein und Perdue 1992; Dietrich; Klein; Noyau 1995). Man kann die Grundgedanken in drei Punkten zusammenfassen:

A. Im Verlauf des Erwerbsprozesses durchläuft der Lerner eine Reihe von Lernervarietäten. Sowohl die interne Organisation einer jeden solchen Varietät zu einem gegebenen Zeitpunkt wie auch der Übergang von einer Varietät zur Folgenden sind durch eine inhärente Systematik gekennzeichnet: eine Lernervarietät ist also ein eigenständiges, mit den angrenzenden Varietäten verwandtes sprachliches System.

B. Es gibt eine beschränkte Anzahl von Organisationsprinzipien, die sich in allen Lernervarietäten finden. Die Struktur einer bestimmten Äußerung in einer Lernervarietät ergibt sich aus dem Zusammenwirken dieser Organisationsprinzipien. Ihr Zusammenspiel variiert in Abhängigkeit von verschiedenen Faktoren, beispielsweise der Ausgangssprache des Lerners, der fortlaufenden Inputanalyse und anderen. Wenn der Lerner beispielsweise dem Input ein neues Element der Nominalmorphologie „entrissen“ hat, so mag dies das Gewicht der verschiedenen anderen Möglichkeiten, ein nominales Argument zu markieren, ändern. Ein neues Morphem, eine neue Konstruktion zu lernen, heißt bei dieser Betrachtungsweise nicht, dass es dem Lerner gelungen ist, einen weiteren Stein in das Puzzle „Zielsprache“ einzufügen, das er zusammenlegen muss. Vielmehr führt es zu einer oft minimalen, bisweilen aber auch substantiellen Umorganisation der gesamten Varietät; dabei gleicht sich die Balance der einzelnen Faktoren allmählich jener an, die für die Zielvarietät charakteristisch ist.

C. Lernervarietäten sind nicht unvollkommene Nachahmungen einer ,eigentlichen Sprache" - nämlich der Zielsprache -, sondern eigenständige, in sich fehlerfreie Systeme, die sich durch ein besonderes lexikalisches Repertoire und besondere morphosyntaktische Regularitäten auszeichnen. Voll entwickelte Sprachen wie Deutsch, Englisch, La- 
tein sind einfach Grenzfälle von Lernervarietäten. Sie repräsentieren einen relativ stabilen Zustand des Spracherwerbs - jenen Zustand, zu dem der Lerner mit seinem Erwerbsprozess aufhört, weil es zwischen seiner Varietät und der Sprache seiner jeweiligen sozialen Umgebung keinen wahrnehmbaren Unterschied mehr gibt.

In dieser Betrachtungsweise sind alle Lernervarietäten, und darunter als Grenzfall auch die ,eigentlichen" Sprachen, Manifestationen der menschlichen Sprachfähigkeit. Wenn man das Wesen dieser Fähigkeit verstehen will und ebendies ist das Ziel der Linguistik - , dann empfiehlt es sich, nicht unbedingt mit den kompliziertesten Fällen, eben den voll ausgebildeten Sprachen, zu beginnen und von diesen auf die elementareren Manifestationen der menschlichen Sprachfähigkeit zurückzublicken. Die Spracherwerbsforschung ist keine Anwendung linguistischer oder psychologischer Einsichten und Begrifflichkeiten auf einen Randbereich - sie ist ein genuiner Beitrag zur Erforschung der menschlichen Sprachfähigkeit.

\section{Abschließende Bemerkung}

Die beiden heute vorherrschenden Paradigmen nehmen viele Züge der älteren Theoriedebatte auf, beispielsweise die Idee der Pidginisierung (Schumann 1978) in der Vorstellung, dass Pidgins bestimmte elementare Lernervarietäten sind, oder die Rolle der Kontrastivität in der Vorstellung, dass Parameter neugesetzt werden müssen. Sie sind in vielen Punkten gegensätzlich, aber nicht in jeder Hinsicht unvereinbar (Eubank 1991). So bewegen sich die Untersuchungen von Meisel und Mitarbeitern zu Erst- und Zweitspracherwerb im generativen Paradigma, aber sie versuchen die Entwicklung verschiedener Varietäten über einen längeren Zeitraum nach ihrer internen Dynamik zu rekonstruieren und zeigen insofern viele Berührungspunkte mit dem Lernervarietäten-Ansatz. Umgekehrt haben Klein und Perdue (1997) gezeigt, dass sich ihre Befunde über die „Grundvarietät" und ihren weiteren Ausbau im Sinne des Minimalismus, der jüngsten Variante der generativen Grammatik, deuten lassen. Es ist daher nicht ausgeschlossen, dass es in der $\mathrm{Zu}$ kunft zu einer Konvergenz dieser beiden Richtungen und damit $\mathrm{zu}$ einer geschlossenen, einheitlichen Theorie des Spracherwerbs kommen wird.

\section{Literatur in Auswahl}

Carroll, Mary; Rainer Dietrich; Günther Storch (1982): Learner Language and Control. Frankfurt.

Chomsky, Noam (1981): Lectures on Government and Binding. Amsterdam.

Corder, Pit (1981): Error Analysis and Interlanguage. Oxford.

Dietrich, Rainer; Wolfgang Klein; Colette Noyau (1995): Temporality in a Second Language. Amsterdam.

Epstein, Samual David; Susan Flynn; Gita Martohardjono (1997): Second Language Acquisition: Theoretical and Experimental Issues in Contemporary Research. In: Behavioural and Brain Sciences 19, 677-758

Ellis, Rod (1994): The Study of Second Language Acquisition. Oxford.

Eubank, Lynn (Hg.) (1991): Universal Grammar in the Second Language. Amsterdam.

Fletcher, Paul; Brian MacWhinney (Hg.) (1995): The Handbook of Child Language. Oxford.

Kellerman, Eric; Mike Sharwood-Smith (Hg.) (1986): Crosslinguistic Influence in Second Language Acquisition. London.

Klein, Wolfgang (1995): Language Acquisition at Different Ages. In: David Magnusson (Hg.): The Lifespan Development of Individuals. Cambridge. 244-264.

-; Clive Perdue (1992): Utterance Structure. Amsterdam.

-; - (1997): The Basic Variety. In: Second Language Research 13, 301-347.

Krashen, Steve (1981): Second Language Acquisition and Second Language Learning. Oxford.

Lenneberg, Eric (1967): Biological Foundations of Language. New York.

MacLaughlin, Barry (1978): Second Language Acquisition in Childhood. Hilldale/N. J.

Meisel, Jürgen (Hg.) (1994): Bilingual First Language Acquisition. Amsterdam.

Meisel, Jürgen; Harald Clahsen; Manfred Pienemann (1981): On determining developmental stages in natural second language acquisition. In: Studies in Second Language Acquisition 3, 109- $\overline{135}$.

Odlin, Terence (1989): Language Transfer. Cambridge.

Perdue, Clive (Hg.) (1993): Adult Language Acquisition: Crosslinguistic Perspectives. Cambridge. (2 Bd.).

Pulvermüller, Friedemann; John Schumann (1994): Neurological Mechanisms of Language Acquisition. In: Language Learning 44, 681-734.

Ritchie, William C.; Tej Bhatia (Hg.) (1996): Handbook of Second Language Acquisition. New York. 
Schumann, John H. (1978): The pidginization process: a model for second language acquisition. Rowley, MA.

Singleton, David (1989): Language Acquisition: the Age Factor. Clevedon.

Slobin, Dan (Hg.) (1985): The Crosslinguistic Study of Language Acquisition. New Jersey. (2 Bd.).

Tropf, Herbert (1983): Variation in der Phonologie des ungesteuerten Spracherwerbs. Phil. Diss. (masch.), Universität Heidelberg.
Weissenborn, Jürgen; Helen Goodluck; Tom Roeper (Hg.) (1992): Theoretical Issues in Language Acquisition. Hillsdale/N. J.

White, Lydia (1989): Universal Grammar and Second Language Acquisition. Amsterdam.

Wode, Henning (1981): Learning a Second Language 1: An Integrated View. Tübingen.

Wolfgang Klein, Nijmegen (Niederlande) 